\title{
Nonlinear, Nonpolar Solvation Dynamics in Water: The Roles of Electrostriction and Solvent Translation in the Breakdown of Linear Response
}

\author{
Damian Aherne, Vu Tran, and Benjamin J. Schwartz* \\ Department of Chemistry and Biochemistry, University of California at Los Angeles, \\ Los Angeles, California 90095-1569 \\ Received: January 27, 2000; In Final Form: April 5, 2000
}

The fact that the motion of solvent molecules defines the reaction coordinate for electron-transfer and other chemical reactions has generated great interest in solvation dynamics, the study of how the solvent responds to changes in a solute's electronic state. In the limit of linear response (LR), when the perturbation caused by the solute is "small", the relaxation of the excited solute's energy gap should behave identically to the relaxation dynamics of the unperturbed solute following a natural fluctuation of the gap away from equilibrium. Despite the fact that the addition of a fundamental unit of charge to a small solute results in a solvation energy that is tens or hundreds of $k T$, computer simulations of solvation dynamics have found, with only a few exceptions, that LR is obeyed for changes in solute charge. Essentially none of this work, however, accounts for the fact that the solutes in real chemical reactions undergo changes in size and shape as well as in charge distribution. In this paper, we compare the results of molecular simulations of polar and nonpolar solvation dynamics for a simple Lennard-Jones solute in a flexible-water solution to explore the validity of LR. We find that, when short-range forces are involved, LR breaks down dramatically: both the inertial and diffusive components of the relaxation differ from those predicted by LR. For increases in solute size, expansion of the solute drives the first-shell solvent molecules into the second shell. The resulting nonequilibrium relaxation takes advantage of translation-rotation coupling that does not occur at equilibrium, resulting in faster solvation than that predicted by LR. Decreases in solute size, on the other hand, result in inward translational motions of solvent molecules that affect the solute's energy gap by destabilizing the energy of the (unoccupied) ground state. The inward motions involved in the nonequilibrium relaxation are not present at equilibrium because the destabilization of the ground state is much larger than $k T$. Because the energetically most important solvent molecules, those closest to the solute, are just as likely to be moving away from the solute as toward it at the time of excitation, solvation for decreases in size is much slower than predicted by LR. In the most realistic cases, when both the size and the charge of the solute change, the solvent translational motions resulting from the size change and those resulting from electrostriction, the net ion-dipole attraction between the charged solute and the polar solvent, combine in an additive fashion. When the solute both gains a charge and expands, the translational motions resulting from electrostriction nearly cancel those from the outward solute expansion so that rotational motions dominate the solvent response; the small net expansion that remains results in only a minor breakdown of LR. The additional inward solvent translations beyond those required by electrostriction, which are necessary when the solute becomes charged and its size decreases, on the other hand, result in a severe breakdown of LR. All of the results are compared with previous experimental and theoretical studies of solvation dynamics, and the implications for solvent-driven chemical reactions are discussed.

\section{Introduction}

The solvent in a chemical reaction is far from being a mere spectator that serves only as a medium to support reactants and products until they can encounter one another by diffusion. Instead, the motions of solvent molecules and the way they arrange themselves around reacting species critically affect the dynamics of chemical reactions in solution. Particularly for electron-transfer reactions, the polarization of the solvent defines the reaction coordinate: the local solvent configuration determines the relative energies of the reactants and products, and the fluctuations determine the rate at which charge transfer occurs. ${ }^{1}$ Thus, there has been an enormous amount of recent interest in solvation dynamics, the study of the response of the

* Corresponding author. E-mail: schwartz@chem.ucla.edu. solvent to changes in the electronic charge distribution of a solute. ${ }^{2}$ At the heart of the matter are the specific solvent motions that lead to relaxation and the ways in which the relative efficiencies of these motions change in response to a particular perturbation of the solute. Although new advances in ultrafast laser technology have allowed great strides to be made in our ability to measure solvent relaxation dynamics via the timedependent Stokes shift or the photon-echo decay of fluorescent probe molecules, it is still impossible for experiments to determine precisely which solvent motions are responsible for relaxation. Thus, most of our insight in identifying specific solvent motions and analyzing solvation dynamics on a molecular level comes from the realm of computer simulations.

Computer simulations of solvation dynamics can be constructed in a straightforward manner. ${ }^{2,3}$ After equilibrating a 
simulated ground-state solute with a simulated solvent, the solute-solvent interaction potential is suddenly altered to reflect a change in the solute's electronic state. This excitation occurs instantaneously on the time scale of solvent nuclear motion, placing the solvent nuclear coordinates out of equilibrium with the solute's excited-state interaction potential. The solvent molecules respond to this perturbation by rearranging their nuclear degrees of freedom to decrease the excited-state solutesolvent potential energy, while at the same time, the solutesolvent potential energy for the original interaction potential increases as the solvent molecules move away from their former, ground-state equilibrium. The net result is that the energy gap between the (unoccupied) ground state and the (occupied) excited state of the solute continuously decreases with time as the solvent responds. The decrease in the solute's energy gap with time is typically used to compute a solvent response function $^{2}$

$$
S(t)=\frac{\bar{U}(t)-\bar{U}(\infty)}{\bar{U}(0)-\bar{U}(\infty)}
$$

where $U(t)=E_{\text {exc }}(t)-E_{\text {gnd }}(t)$ is the solute's energy gap at time $t$ and the overbar denotes a nonequilibrium ensemble average. The solvent response function $S(t)$ is normalized to start at unity at time zero and to decay to 0 as time approaches infinity, providing a convenient method for comparing solvent responses for different solute/solvent combinations.

Nonequilibrium molecular simulation trajectories contain a complete record of the motions of each individual solvent molecule, offering the possibility for a detailed molecular analysis of the solvent motions involved in relaxation. This type of detailed information comes at the price, however, of requiring significant computational resources. A nonequilibrium ensemble average first requires a long equilibrium molecular dynamics or Monte Carlo simulation to be run to generate a series of uncorrelated equilibrium solvent configurations from which the nonequilibrium trajectories can be launched. From these starting configurations, tens or usually hundreds of excited-state trajectories must be calculated in order to provide decent statistics for nonequilibrium ensemble properties, such as the time variation of the solute's energy gap following excitation. Moreover, to make comparisons between the dynamics following different perturbations (for example, ionizing the solute versus changing its dipole moment), an entirely new set of nonequilibrium trajectories must be computed for each perturbation. These computational requirements can be significantly reduced, however, by taking advantage of the linear response (LR) approximation. The LR approximation relies on the Onsager regression hypothesis, which states that, for a small enough perturbation, the relaxation of a perturbed solute's energy gap back to equilibrium should behave identically to the relaxation dynamics of the unperturbed solute following a natural fluctuation of the gap away from equilibrium. ${ }^{4}$ Thus, in the limit of LR, the equilibrium solvent response function

$$
C(t)=\frac{\langle\delta U(0) \cdot \delta U(t)\rangle}{\left\langle(\delta U)^{2}\right\rangle}
$$

should decay identically to the nonequilibrium response function $S(t)$, eq $1 .^{2}$ In eq $2, \delta U(t)=U(t)-\langle U\rangle$ is the fluctuation of the energy gap from its equilibrium value, and the angled brackets denote an equilibrium ensemble average. The use of eq 2 to compute the solvent response function provides an enormous computational advantage in that the same equilibrium run can be used to compute the solvent response for a variety of perturbations. This is true because the computationally expensive forces never need to be computed using the excited-state interaction potential, so the energy gaps for any number of desired perturbations can be computed simultaneously from a trajectory propagated using forces only from the ground-state solute-solvent interaction. Thus, instead of the multiple sets of hundreds of trajectories needed to compare nonequilibrium solvation dynamics, use of the LR approximation reduces the computational requirements for multiple solute perturbations to a single ground-state trajectory.

Given the computational savings in the use of the LR approximation, it would seem almost unfair to be able to accurately predict the dynamics of a variety of solute perturbations from only a single equilibrium trajectory using eq 2 . Yet, in nearly every computer simulation study of solvation dynamics (but not all ${ }^{5-8}$ ), the LR assumption of eq 2 is able to predict, remarkably well, the majority of the nonequilibrium response, eq 1 , when the solute undergoes a change in charge distribution. ${ }^{9-16}$ The agreement is good enough that many recent studies have chosen to forego the computation of nonequilibrium trajectories and base their conclusions solely on predictions from the LR approximation. ${ }^{17-25}$ In a recent paper, we explored the LR predictions for solvation dynamics in water not just for changes in charge but also for changes in solute size, shape, and polarizability. ${ }^{20} \mathrm{We}$ found that the short-range forces involved in these latter types of perturbations caused the bulk of the solute-solvent relaxation energy to be carried by only the one or two closest solvent molecules. This led to a nonlinear variation of the solute energy gap with solute size and produced equilibrium solvent response functions (eq 2) for different size changes that were not identical. Moreover, whereas the relaxation dynamics for the appearance of either a positive or a negative charge on the solute were identical, for combined changes in both solute size and charge, the resulting relaxation depended sensitively on both the magnitude and the sign of both the size and the charge changes. ${ }^{20}$ All of these results suggest that, when the perturbation involves short-range forces, the LR approximation is likely to fail.

In this paper, we explore the nature of the linear response approximation in detail by comparing the equilibrium and nonequilibrium solvent dynamics of water following a variety of solute size and size-and-charge changes. In electron-transfer and other reactions, the reactant's size and polarizability can change as well as the charge. Thus, for this work, we have chosen perturbations that are designed to mimic those experienced by real solutes undergoing electron-transfer reactions by combining size and charge changes. Like previous studies, ${ }^{9-16}$ we find that, when the perturbation involves only a change in charge, the same solvent motions cause relaxation both at equilibrium and during nonequilibrium dynamics. For solute size changes, on the other hand, we identify solvent translations as the key motions that differ between the equilibrium and nonequilibrium dynamics, leading to a breakdown of LR. For combined size-and-charge changes, we find that there is an interplay between the solvent translations driven by the size change and those driven by electrostriction, the net ion-dipole attraction between the newly charged solute and the solvent. These translational motions can act either cooperatively or antagonistically; the net result is that either translational or rotational solvent motions can dominate the overall relaxation dynamics depending on the specific nature of the perturbation.

Because of the importance of short-range forces in the breakdown of LR, we make, in this paper, a somewhat artificial distinction between two classes of solvation: dielectric (or polar) 
solvation, primarily composed of solvent rotational motions in response to reactant charge changes, and mechanical (or nonpolar) solvation, comprising the translational solvent motions that couple to reactant changes in size or polarizability. For dielectric solvation, the time evolution of the relaxation is well understood and is usually classified into three regimes. First, an initial Gaussian response results from the inertial rotational motions of solvent molecules in the first solvation shell; ${ }^{14,26}$ in polar solvents such as water ${ }^{9,10,15,16,27}$ or acetonitrile, ${ }^{13,28}$ this inertial component can account for $60-80 \%$ of the total solvent relaxation. After the inertial response is complete, a subsequent librational relaxation occurs, characterized by rapid, damped oscillatory solvent rotational motions. ${ }^{9,13}$ Finally, the remainder of the relaxation occurs on a slower, diffusive time scale that often matches well with the relaxation time(s) predicted by dielectric continuum theories. ${ }^{2}$ The same types of librational solvent motions involved in the nonequilibrium relaxation are also present in the equilibrium dynamics, so dielectric relaxation usually falls within the LR regime.

Mechanical relaxation, on the other hand, has been studied much less than the more traditional dielectric solvation dynamics. Only recently have viscoelastic continuum theories been developed as a nonpolar counterpart to dielectric continuum theories. ${ }^{29}$ Experiments in this area are also just beginning, ${ }^{30}$ in large part because it is difficult to find solutes that undergo large size or shape changes without also undergoing a simultaneous change in charge distribution. Moreover, in many solvents, dielectric and mechanical relaxation theories predict similar solvent responses, making an experimental distinction between the two somewhat difficult. ${ }^{31}$ Perhaps most surprisingly, practically every computer simulation done to date has explored the solvent response to changes in solute charge distribution without an accompanying change in size, shape, or polarizability; there have been only a limited number of simulations that have explored nonpolar solvation dynamics. ${ }^{12,20-25,32}$ In our previous paper, we found that, even in a highly polar solvent like water, the solvation energy accompanying solute size changes could be comparable to that induced by solute charge changes, placing polar and nonpolar solvation on an equal energetic footing. ${ }^{20}$ The solvent response to solute size changes consisted primarily of translational motions of the closest one or two solvent molecules. This produces nonpolar relaxation dynamics that are much slower than the corresponding librational response in dielectric solvation and suggests that continuum theories of nonpolar solvation that ignore the molecular nature of the solvent are likely to fail. Finally, as mentioned above, for the most realistic case of a simultaneous change in both size and charge, the two types of solvent response interact in a nonlinear fashion so that either translational or rotational motions can dominate the overall relaxation depending on the particular size/charge perturbation involved. ${ }^{20}$

It is important to note that only a few of the simulations that included nonpolar solvation effects have investigated nonequilibrium dynamics. ${ }^{12,22}$ The bulk of the simulation research on nonpolar solvation, ${ }^{21,23-25}$ including our own previous work, ${ }^{20}$ has relied on the LR approximation to calculate the relaxation dynamics. Given all of the above indications that LR breaks down for the case of nonpolar solvation, many of the conclusions drawn from these studies may not be valid. Thus, we spend the remainder of this paper exploring nonequilibrium, nonpolar solvation dynamics, with a particular emphasis on identifying the reasons for the breakdown of LR. In section II, we present some of the details of the computational algorithms and models used for our simulations. We then start section III.A with a detailed exploration of the LR approximation for mechanical solute perturbations. We find that LR fails for solute size changes, and we identify solvent translational motions as key to the disagreement between the equilibrium and nonequilibrium dynamics. Section III.B goes on to explore the nature of LR for the class of perturbations expected to be most relevant to charge-transfer reactions, that of a simultaneous change in both solute size and charge. Although the response to changes in charge alone is nearly linear, the interplay between solvent translational motions resulting from the size change and those resulting from electrostriction produces a striking nonlinearity for combined changes in size and charge. We take advantage of the molecular detail offered by nonequilibrium simulations in section III.C by exploring the dynamics of the local solvation structure following solute size-and-charge changes via radial and angular pair distribution functions. Finally, we conclude in section IV by pointing out that, even in polar fluids such as water, the breakdown of LR for nonpolar solvation has important implications for charge-transfer and other solution-phase reactions.

\section{Methods}

In addition to the fact that there are few solvents as important in chemistry as water, the choice of water for our solvation dynamics simulations has the additional advantage of allowing us to use spectral density analysis to identify the specific solvent motions involved in relaxation. The time-dependent (equilibrium or nonequilibrium) energy gap $U(t)$ underlying the solvent response functions introduced in eqs 1 and 2 above can be easily Fourier transformed.

$$
I(\omega)=\left|\frac{1}{\sqrt{2 \pi}} \int_{-\infty}^{\infty} \mathrm{d} t \mathrm{e}^{-\mathrm{i} \omega t}[U(t)-\langle U\rangle]\right|^{2}
$$

The resulting spectral density, $I(\omega)$, serves as a histogram of the solvent molecular motions that participate in the relaxation, tabulated by frequency. ${ }^{20}$ Assigning specific molecular motions to each frequency that appears in this type of plot, which is known as the "solvation spectrum" or "influence spectrum", is usually quite challenging because many of the observed motions are intermolecular in nature. Water is unique, however, in that it has been so well studied by both experiment and theory that it is possible to assign particular solvent motions to each frequency that occurs in the influence spectrum. ${ }^{33}$ The SPC flexible-water model ${ }^{34}$ used in our simulations is characterized by high-frequency intramolecular $\mathrm{O}-\mathrm{H}$ stretching (3200-4000 $\mathrm{cm}^{-1}$ ) and bending motions $\left(1800-2000 \mathrm{~cm}^{-1}\right)$, as well as lower-frequency intermolecular librational $\left(\sim 400-1000 \mathrm{~cm}^{-1}\right)$ and hindered translational motions $\left(0\right.$ to $\left.\sim 400 \mathrm{~cm}^{-1}\right)$. These lower-frequency motions can be further distinguished as intermolecular H-bond stretching motions $\left(\sim 300 \mathrm{~cm}^{-1}\right)$ and $\mathrm{H}$-bond bending motions $\left(\leq 200 \mathrm{~cm}^{-1}\right){ }^{33}$ Thus, an examination of the frequencies that appear in the influence spectrum will allow us to directly assign the particular solvent motions involved in both the equilibrium and the nonequilibrium relaxation dynamics.

The computational details of the simulations presented here are essentially identical to those reported in our previous work that studied the equilibrium solvation dynamics of water for nonpolar solute changes. ${ }^{20}$ Our simulated water uses the flexible simple point charge model (SPC/F) due to Toukan and Rahman. ${ }^{34}$ In SPC/F, intramolecular flexibility is grafted onto the well-known SPC intermolecular potential, ${ }^{35}$ which consists of Lennard-Jones (L-J) interactions centered on oxygen sites plus Coulomb interactions between partial charges located at both 
the oxygen and the hydrogen sites. The properties of this model of water have been explored in detail and compared both to other models and to experiment. ${ }^{36}$

The model system in our simulations consists of a cubic cell $24.66 \AA$ on each side containing 500 classical SPC/F molecules (solvent density of $0.997 \mathrm{~g} / \mathrm{mL}$ ) and one solute atom, which has the same mass as an oxygen atom. For the solute ground state, the water-solute interaction was chosen to be identical to the water-water L-J potential

$$
V_{\mathrm{L}-\mathrm{J}}=4 \epsilon\left[\left(\frac{\sigma}{r}\right)^{12}-\left(\frac{\sigma}{r}\right)^{6}\right]
$$

with the same values for the Lennard-Jones $\sigma$ and $\epsilon$ parameters. Thus, our ground-state solute behaves essentially as an uncharged oxygen atom, similar to the "small" solute used by Maroncelli and Fleming in their simulation study of dielectric solvation in water. ${ }^{9}$ The various solute excited states in our simulations were constructed simply: by changing $\sigma$ (changing size) in eq 4; by adding a charge to the solute, which interacts with the solvent charges via the Coulomb potential; or by combinations of both of these changes. Of course, electronic excitation of a solute can also change the well depth of the solute-solvent interaction potential (the L-J $\epsilon$ parameter); ${ }^{12,20,22,23,25}$ for the discussion here, however, we will focus solely on mechanical perturbations in the solute size. The solute's energy gap was computed by calculating the difference in solvation energy (the potential energy of interaction between the solute and all of the water molecules in the simulation) between the ground- and excited-state interaction potentials. The simulations employ standard periodic boundary conditions, and all interactions were evaluated with a smooth spherical cutoff, ${ }^{37}$ terminating at a distance of $12 \AA$. The equations of motion were integrated using the Verlet algorithm with a 0.5 -fs time step in the microcanonical ensemble. ${ }^{3}$ Total energy in the simulations was conserved to better than $0.1 \%$.

The starting point for the equilibrium simulation was obtained by injecting the L-J solute into an equilibrated configuration for neat water. The combined solute-solvent system was equilibrated with velocity rescaling for $10 \mathrm{ps}$ to enforce an average temperature of $298 \pm 10 \mathrm{~K}$ and then equilibrated for an additional 20 ps without rescaling. All of the equilibrium ensemble results were averaged over a subsequent 100-ps ground-state trajectory. ${ }^{20}$ For this study, to best simulate experimental conditions in which the solute is excited just when its energy gap is resonant with a particular laser frequency, we chose to launch nonequilibrium trajectories only when the equilibrium gap energy was within $5 \%$ of its average value. ${ }^{38}$ Thus, we broke the ground-state trajectory into 100 equal intervals and started each of the 100 nonequilibrium trajectories from the first configuration in each interval that had a gap energy equal to that of the "excitation laser". The $\sim 1-p s$ interval between starting configurations ensures that the different nonequilibrium trajectories are uncorrelated. The nonequilibrium dynamics were followed for $2 \mathrm{ps}$. At the end of the nonequilibrium runs, the system showed a temperature rise of $\sim 10 \mathrm{~K}$ for size-only changes and $\sim 25 \mathrm{~K}$ for charge-only or combined charge-and-size changes.

\section{Breakdown of Linear Response for Nonpolar Solvation}

When a neutral solute is ionized in aqueous solution, the solvation energy of the newly formed ion can be 1 or 2 orders of magnitude larger than $k T$ at room temperature. Given that the LR approximation should hold only for small perturbations (so that the relaxation from the perturbation involves the same solvent motions that are present at equilibrium), why does the LR approximation work as well as it does for solute charge changes? One argument is that, because the Coulomb force responsible for dielectric relaxation is long-range, there are a wide variety of fluctuations throughout the solvent that couple to the change in the solute's charge distribution. If the number of molecules that couple is large, then the central limit theorem dictates that the force fluctuations will be Gaussian in nature, thereby leading to LR. Thus, the fact that the majority of the relaxation for solute size changes is caused by just the few closest solvent molecules suggests that the LR approximation is likely to fail for nonpolar solvation. ${ }^{20}$

Although nearly every simulation of dielectric relaxation (changes in charge only) has found that LR is obeyed, Fonseca and Ladanyi have found that LR breaks down in methanol. ${ }^{5}$ In their simulations, Fonseca and Ladanyi modeled a dumbbellshaped solute that starts with a dipolar ground state and has the dipole reversed in the excited state. These authors found that the LR prediction agreed well with the nonequilibrium dynamics during the inertial regime, but that the LR approximation failed during the subsequent relaxation. Fonseca and Ladanyi reasoned that the good initial agreement reflects the fact that the inertial motions of the proximal $\mathrm{O}-\mathrm{H}$ groups at equilibrium are the same motions that bring about the initial nonequilibrium relaxation, leading to LR for the inertial dynamics. ${ }^{5}$ These inertial motions disrupt the equilibrium solvent H-bond structure around the solute; buildup of the new solvent structure for the excited-state solute requires slower solvent motions that are not present at equilibrium, leading to a breakdown of LR on longer time scales. Fonseca and Ladanyi also argued that the reason LR holds well for dielectric solvation in solvents such as water $^{9,10,16}$ or acetonitrile ${ }^{13}$ is that these solvents have an inertial component that accounts for $60-80 \%$ of the total solvent response, so that the LR approximation works well for the majority of the relaxation. ${ }^{5}$ Because methanol has only one small moment of inertia, only a small fraction $(\sim 10 \%)$ of the relaxation is accomplished by the inertial dynamics, leading to the observed breakdown of LR on longer time scales.

A. Translational Phase and the Breakdown of Linear Response for Solute Size Changes. In this section, we will argue that, for nonpolar solvation in water, the reasons for the breakdown of LR are quite different from those observed by Fonseca and Ladanyi for polar solvation in methanol. We will show not only that LR does not describe the dynamics for longtime relaxation following size changes, but also that LR fails during the inertial regime. In our previous paper, we found that the (LR-predicted) amplitude of the inertial component for nonpolar solvation is $\geq 60 \%$ of the total relaxation, similar to that for polar solvation. ${ }^{20}$ Thus, the reason for the breakdown of LR is not the lack of effectiveness of inertial translational motions in causing relaxation, as would be suggested by previous work. ${ }^{5-8}$ Rather, the LR approximation fails because the perturbations in nonpolar solvation are extremely shortrange. What we will argue is that, with only a few molecules strongly coupled, the inertial solvent translations that are present at equilibrium are not the motions that cause relaxation during nonequilibrium dynamics, leading to the failure of LR for nonpolar solvation dynamics.

How badly does LR fail for nonpolar solvation dynamics? Figure 1 compares the nonequilibrium solvent response function $S(t)$, computed via eq 1 (dotted curves), to the equilibrium solvent response $C(t)$, calculated from the ground-state dynamics via eq 2 (solid curves; same as those shown in Figure 4 of our 


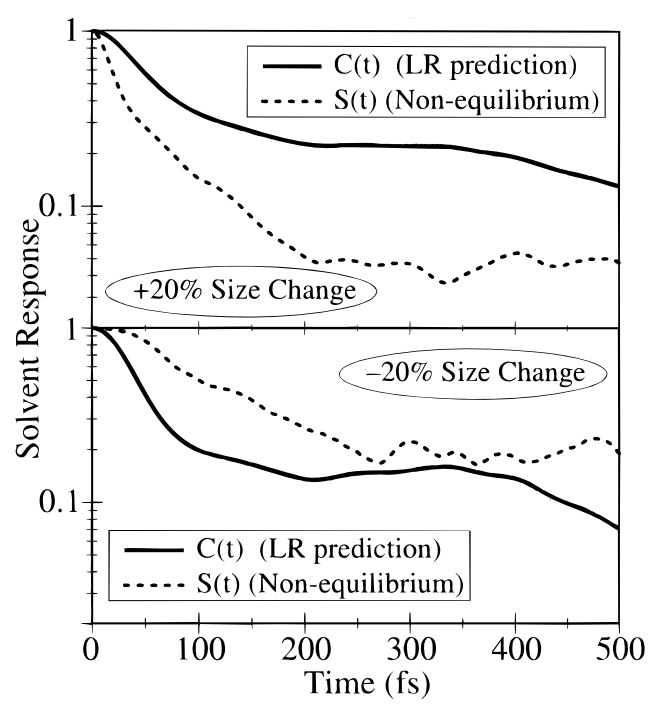

Figure 1. Semilog plot of solvent response functions for a LennardJones solute in SPC/F water for two different nonpolar solute perturbations: a 20\% increase in solute size (upper panel) and a $20 \%$ decrease in solute size (lower panel). The magnitude of the size perturbation refers to the percentage change in the Lennard-Jones $\sigma$ parameter (eq 4). The solid curves show the LR prediction, $C(t)$, from eq 2 ; the dotted curves are the nonequilibrium relaxation, $S(t)$, from eq 1.

previous paper ${ }^{20}$ ). The upper and lower panels show the solvent response functions for a $20 \%$ solute size increase and decrease, respectively. The strong disagreement between the two sets of curves indicates that LR does not hold for either size increases or size decreases. To better characterize the data, we fit all of the solvent response functions presented in this paper to a Gaussian plus exponential decay, with fit parameters summarized in Table 1. As is clear from both the figure and the table, not only does the LR prediction fail for the long-time relaxation, but the inertial relaxation times differ from those expected from LR by over a factor of 2 . Even more surprising is the fact that, for the size increase, the nonequilibrium solvation dynamics are considerably faster than those predicted by LR but, for the size decrease, the nonequilibrium dynamics are substantially slower than the LR prediction.

How can we account for the large differences between the equilibrium and nonequilibrium solvent dynamics in Figure 1? Figure 2 shows the influence spectra underlying the solvent relaxation for both the equilibrium (solid curves) and nonequilibrium (dotted curves) solvent relaxation, computed via eq 3; as with Figure 1, the upper panel is for the $+20 \%$ size change, and the lower panel is for the $-20 \%$ size change. We find that intramolecular solvent motions do not contribute to nonpolar relaxation, so the influence spectra presented in Figure 2 are shown only for frequencies up to $1200 \mathrm{~cm}^{-1}$. We also expect that solvent librational motions will not be present in the influence spectrum for solute size changes because there are no direct interactions between the nonpolar solute and the solvent $\mathrm{H}$ atoms. ${ }^{20}$

The solid curve in the upper panel of Figure 2 shows the solvent motions present at equilibrium that strongly affect the energy gap for the solute whose excited state is $20 \%$ larger than the ground state. As discussed in our previous paper, ${ }^{20}$ the most important solvent motion for modulating the solute's energy gap at equilibrium is the intermolecular $\mathrm{H}$-bond stretch near $300 \mathrm{~cm}^{-1}$; this is fastest motion available for the translation of an entire water molecule. Lower-frequency intermolecular motions also contribute, but as expected, there are essentially

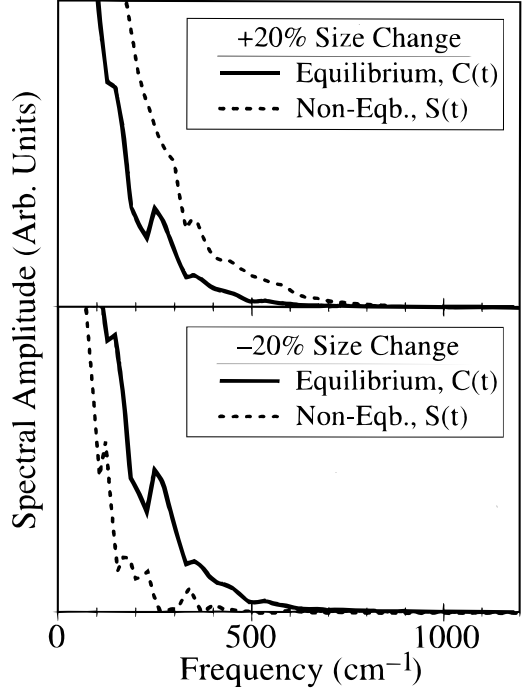

Figure 2. Influence spectra (eq 3 ) for both equilibrium (solid curves) and nonequilibrium (dotted curves) solvation dynamics for a $20 \%$ solute size increase (upper panel) and a 20\% solute size decrease (lower panel).

no contributions from solvent librations in the frequency region above $400 \mathrm{~cm}^{-1}$. The nonequilibrium dynamics shown by the dotted curve, however, not only have a higher amplitude of the same low-frequency translational motions seen in the equilibrium dynamics but also have a significant contribution from $400-600 \mathrm{~cm}^{-1}$ librational motions. This means that solvent librational motions, which do not affect the energy gap at equilibrium, play an important part in the nonequilibrium relaxation. It is the presence of these higher-frequency motions that leads to the overall faster nonequilibrium relaxation for the solute size increase seen in Figure 1.

Given that the solvent $\mathrm{H}$ atoms do not directly interact with the solute, how can solvent librational motions affect relaxation of the nonpolar excited-state solute? The answer lies in translation-rotation coupling. At equilibrium, the intermolecular $\mathrm{H}$-bond stretching motions of the first-shell solvent molecules strongly modulate the solute's energy gap, but the amplitude of these motions away from the solute is restricted by the presence of the second solvation shell. When the solute is excited, the closest solvent molecules find themselves on a highly repulsive part of the solute-solvent interaction potential. This means that there is a large force driving the closest molecules outward to reestablish equilibrium. The amplitude of the outward motions needed to cause relaxation is larger than the translational fluctuations that are typically present at equilibrium. The first-shell molecules, however, cannot simply increase the amplitude of their $\mathrm{H}$-bond stretching motions because they are hindered by the presence of the second solvent shell. When driven by the steeply repulsive potential, however, these first-shell molecules can increase their outward motion to better accommodate the larger excited-state solute by reorienting (librating) to better fit between the molecules of the second shell. Thus, translation-rotation coupling, which is not present at equilibrium, increases the effectiveness of the translational motions needed to relax the excited-state solute. The signatures of this coupling are the presence of librational motions and the increased amplitude of the low-frequency translational motions in the nonequilibrium influence spectrum in the upper panel of Figure 2.

In the lower panel of Figure 2, the solid curve shows the solvent motions present at equilibrium that strongly affect the energy gap for the solute whose excited state is $20 \%$ smaller 
TABLE 1: Gaussian Plus Exponential Fit Parameters to Solvent Response Functions

\begin{tabular}{|c|c|c|c|c|c|}
\hline solute perturbation & $C(t)$ or $S(t)^{a}$ & $\begin{array}{c}\text { Gaussian } \\
\text { frequency }\left(\mathrm{ps}^{-1}\right)\end{array}$ & $\begin{array}{l}\text { Gaussian } \\
\text { amplitude }\end{array}$ & $\begin{array}{c}\text { exponential } \\
\text { decay time (ps) }\end{array}$ & $\begin{array}{l}\text { relevant } \\
\text { figure }\end{array}$ \\
\hline$+20 \%$ size change & $C(t)$ & 19 & 0.65 & 575 & 1 \\
\hline$+20 \%$ size change & $S(t)$ & 43 & 0.68 & 148 & 1 \\
\hline$-20 \%$ size change & $C(t)$ & 23 & 0.79 & 680 & 1 \\
\hline$-20 \%$ size change & $S(t)$ & 10 & 0.69 & 870 & 1 \\
\hline+1 charge change & $C(t)$ & 104 & 0.62 & 128 & 4 \\
\hline+1 charge change & $S(t)$ & 99 & 0.57 & 270 & 4 \\
\hline+1 charge $/-20 \%$ size change & $C(t)$ & 104 & 0.62 & 132 & 4 \\
\hline+1 charge $/-20 \%$ size change & $S(t)$ & 15 & 0.32 & 170 & 4 \\
\hline-1 charge $/+20 \%$ size change & $C(t)$ & 70 & 0.53 & 140 & 4 \\
\hline-1 charge $/+20 \%$ size change & $S(t)$ & 95 & 0.61 & 213 & 4 \\
\hline
\end{tabular}

${ }^{a}$ Equilibrium responses, $C(t)$, calculated from eq 2; nonequilibrium responses, $S(t)$, calculated from eq 1.

than the ground state, while the dotted curve shows the motions that cause the nonequilibrium relaxation. The solid curve indicates that the same solvent $\mathrm{H}$-bond stretching motions that modulate the equilibrium energy gap for the size increase are also effective at equilibrium for the size decrease. The influence spectrum for the nonequilibrium dynamics, however, shows no sign of the $300 \mathrm{~cm}^{-1} \mathrm{H}$-bond stretching motion; the relaxation is caused solely by low-frequency motions below $200 \mathrm{~cm}^{-1}$. This means that the inertial translational motions of the closest molecules that modulate the energy gap at equilibrium are not able to lower the energy of the excited-state solute following the size decrease. It is the absence of these relatively highfrequency $\mathrm{H}$-bond stretching motions that leads to nonequilibrium relaxation dynamics that are much slower than those predicted by LR, as seen in Figure 1.

Why is the intermolecular solvent H-bond stretching motion, which so effectively modulates the solute's energy gap at equilibrium, unable to participate in the nonequilibrium relaxation? The reason is that the phase of the inertial translational solvent motions becomes important when the steeply repulsive interaction with the solute is removed upon excitation. At equilibrium, the first-shell solvent molecules translate back and forth between the repulsive potentials of the solute and the second solvent shell. When the solute contracts, however, there is no driving force pushing the closest solvent molecules inward to reestablish equilibrium. If the closest solvent molecules happen to be moving away from the solute at the time of excitation, they will continue their outward inertial motion until they encounter the repulsion of the second-shell molecules, delaying the inward motion necessary for relaxation for an entire period of the $\mathrm{H}$-bond stretch. This concept is illustrated in Figure 3 , which shows the early-time solvation dynamics for two individual nonequilibrium trajectories. The dot-dashed curve shows $S(t)$ for a trajectory for which the closest solvent molecule happened to be moving toward the solute $(\mathbf{v} \cdot \mathbf{r}<0)$ at time zero. The inward motion of this molecule continues after excitation of the solute, leading to a Gaussian inertial relaxation on the H-bond stretching time scale. This motion is also present at equilibrium, and indeed, the inertial portion of this nonequilibrium solvent response function resembles that of the equilibrium response function presented in the lower panel of Figure 1.

The dashed curve in Figure 3, on the other hand, shows the nonequilibrium solvent response for a trajectory for which the closest solvent molecule happened to be moving away from the solute $(\mathbf{v} \cdot \mathbf{r}>0)$ at the time of excitation. The inertial motion continues in the outward direction, so no relaxation occurs until either an entire cycle of the translational motion is complete or a lower-frequency motion takes place to allow a different solvent molecule to become the closest to the solute (Figure 3, inset). Statistically, half the trajectories will have the closest molecule

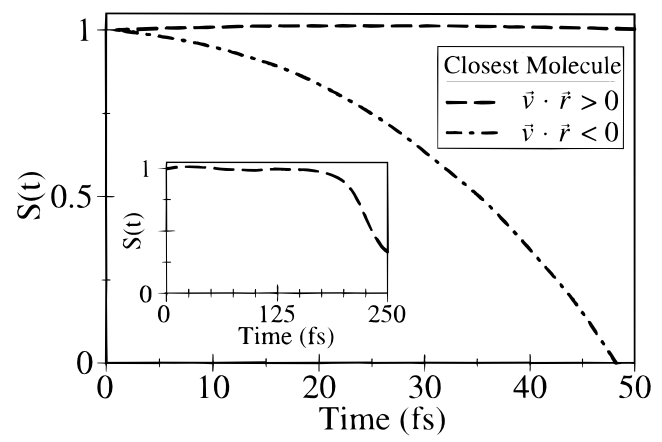

Figure 3. Nonequilibrium solvent response functions, eq 1, computed for two individual trajectories following a $20 \%$ decrease in solute size. The dashed curve shows the solvent relaxation for a trajectory in which the closest solvent molecule was moving away from the solute at the time of excitation $(v \cdot r>0)$. The dot-dashed curve shows the solvent response for a trajectory in which the closest solvent molecule was moving toward the solute at the time of excitation $(v \cdot r<0)$. Inset: $S(t)$ for the same $v \cdot r>0$ trajectory as in the main figure but on a longer time scale.

moving toward the solute, and half will have the closest molecule moving away from the solute at the time of excitation. Thus, the full nonequilibrium average $S(t)$ presented in Figure 1 shows relaxation dynamics roughly halfway between the two curves presented in Figure 3. Overall, because the few solvent molecules that can cause relaxation start their inertial motions with a random phase, the $\mathrm{H}$-bond stretching motion, on average, is ineffective for promoting relaxation following the solute size decrease. This leads to the disappearance of the H-bond stretching feature in the influence spectrum in Figure 2, resulting in the dominance of much lower-frequency motions in the slow nonequilibrium solvation dynamics in Figure 1.

B. Interaction of Translational Motions from Size Changes and Electrostriction. Although the data in Figures $1-3$ provide a great deal of insight into the molecular motions accompanying mechanical solvation, the underlying premise of a solute that changes only its size and not its charge distribution is not very realistic. The change in the electronic wave function of most solutes upon excitation usually results in a combination of a change in dipole moment (or at least a change in quadrupole moment ${ }^{32}$ ) and a change in size, the latter because of the alteration in Pauli repulsion forces and/or molecular polarizability. For aromatic solutes, the effective change in size upon excitation can be on the order of $\sim 10 \% .{ }^{39}$ For the solutes involved in charge-transfer reactions that undergo a change in oxidation state, the addition or removal of electrons can make the effective change in size even larger. ${ }^{40}$ Thus, the most realistic case to consider is that of a solute that undergoes a simultaneous change in both size and charge; modeling only the change in charge or size misses a great deal of the essential physics. In 


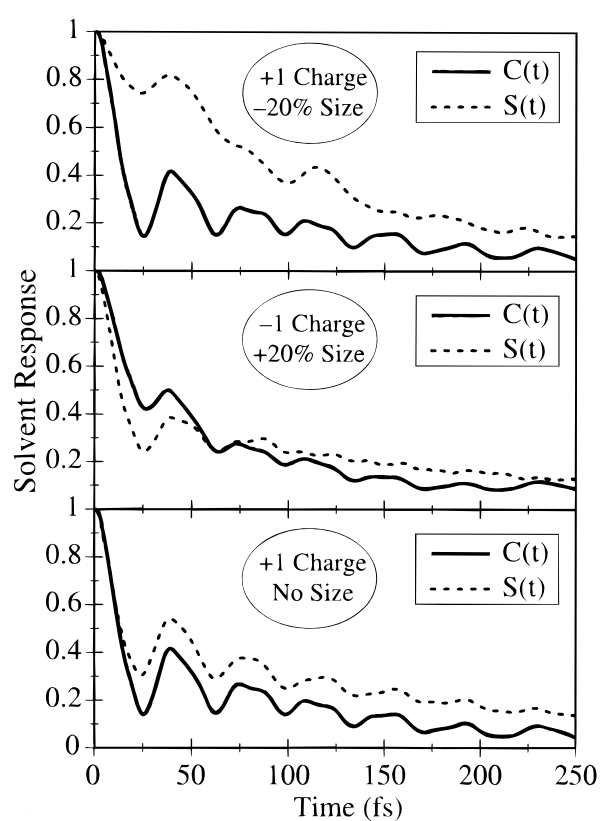

Figure 4. Solvent response functions for a variety of solute perturbations: positive change in charge with a simultaneous $20 \%$ size decrease (upper panel); negative change in charge with a simultaneous $20 \%$ size increase (center panel); and positive change in charge with no change in size (lower panel). The solid curves show the LR prediction, $C(t)$, from eq 2; the dotted curves are the nonequilibrium relaxation, $S(t)$, from eq 1.

our previous paper, we explored the equilibrium solvent dynamics for coupled size-and-charge changes and found that the nature of the solvent response depends on both the magnitude and the sign of the size and charge changes involved. ${ }^{20}$ Given that we have just shown that there is a strong nonlinearity associated with solute size changes, the nonequilibrium solvent response to combined size-and-charge changes is likely to be quite different from that predicted in our previous paper using LR. ${ }^{20}$ Thus, in this section, we explore the way in which the nonequilibrium solvation dynamics for solute size and charge changes couple together.

How well does LR work for combined size-and-charge changes? Figure 4 compares the equilibrium solvent response function $C(t)$ (solid curves; same as in Figure 6 of our previous paper $^{20}$ ) to the nonequilibrium solvent response $S(t)$ (dotted curves) for two realistic cases: that of a neutral solute that is ionized, resulting in a $20 \%$ size decrease (upper panel), and that of a neutral solute that gains an electron, resulting in a $20 \%$ size increase (center panel). The lower panel shows the equilibrium (solid curve) and nonequilibrium (dotted curve) solvent response functions for the case explored by most previous simulation studies: ${ }^{5-11,13-15}$ that of a change in solute charge without an accompanying change in size. For this latter case, other than slightly overestimating the magnitude of the inertial component, LR does an excellent job of predicting the nonequilibrium solvent response (Table 1), in agreement with the previous work of Maroncelli and Fleming. ${ }^{9}$ When the same +1 charge change is combined with a $-20 \%$ size change, however, the upper panel makes clear that LR fails dramatically. For the nonequilibrium relaxation of the combined charge/size decrease, the inertial amplitude, inertial frequency, and subsequent relaxation decay rate all decrease significantly relative to the LR prediction (Table 1). The breakdown of LR is more subtle for the complementary case of a -1 charge change combined with a $20 \%$ size increase, as shown in the center panel. For this case, the two solvent response curves appear to be in

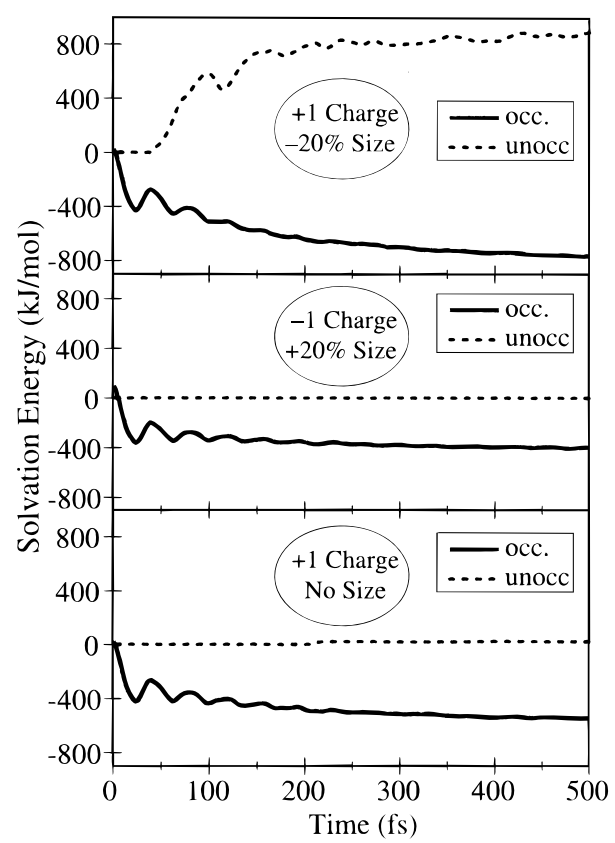

Figure 5. Nonequilibrium dynamical history of the individual unoccupied ground (dashed curves) and occupied excited (solid curves) energy levels for a variety of solute perturbations: positive change in charge with a simultaneous $20 \%$ size decrease (upper panel); negative change in charge with a simultaneous $20 \%$ size increase (center panel); change in charge with no change in size (lower panel). The difference between the two curves in each panel is the energy gap used to compute the nonequilibrium solvent response functions shown in Figure 4.

roughly good agreement and to have similar inertial amplitudes, but the inertial frequency of the nonequilibrium solvent response increases by $35 \%$ and the diffusive relaxation time increases by a factor of 2 relative to the LR prediction (Table 1).

The data in Figure 4 present some interesting challenges in terms of understanding the breakdown of LR. First, why do the inertial frequency and amplitude decrease relative to the LR prediction for size decreases but increase relative to the LR prediction for size increases? Part of this effect is that the nonlinearity observed above for size-only changes carries through to the case of combined size-and-charge changes. The differences between $S(t)$ and $C(t)$ in Figure 4, however, are not the same as those seen for the size-only changes in Figure 1, indicating that the mechanical and dielectric solvent responses must be coupled together. Second, given the energetics involved, why is the LR prediction for solute size decreases so much poorer than that for size increases? Our expectation would have been that the nonlinearity associated with the size decrease would play only a minor role in the combined size-and-charge change, because the solvation energy associated with a $-20 \%$ size change is much smaller than that associated with a +1 charge change. ${ }^{20} \mathrm{We}$ also would have expected the nonlinearity to be more important when the size increases, because the solvation energies associated with each of the $+20 \%$ size and -1 charge changes are comparable. ${ }^{20}$ Instead, Figure 4 shows the reverse to be true, again indicative of a coupling between the solvent motions responsible for polar and nonpolar solvation.

Insight into the nature of the coupling between the dielectric and mechanical solvation responses can be found by investigating the dynamics of the individual energy levels (not the gap) of the solute following excitation, shown in Figure 5, and their corresponding influence spectra, shown in Figure 6. The solid curves in each panel of Figures 5 and 6 display the evolution of the solvation energy of the (occupied) excited-state solute for the same three perturbations shown in Figure 4. The dotted 


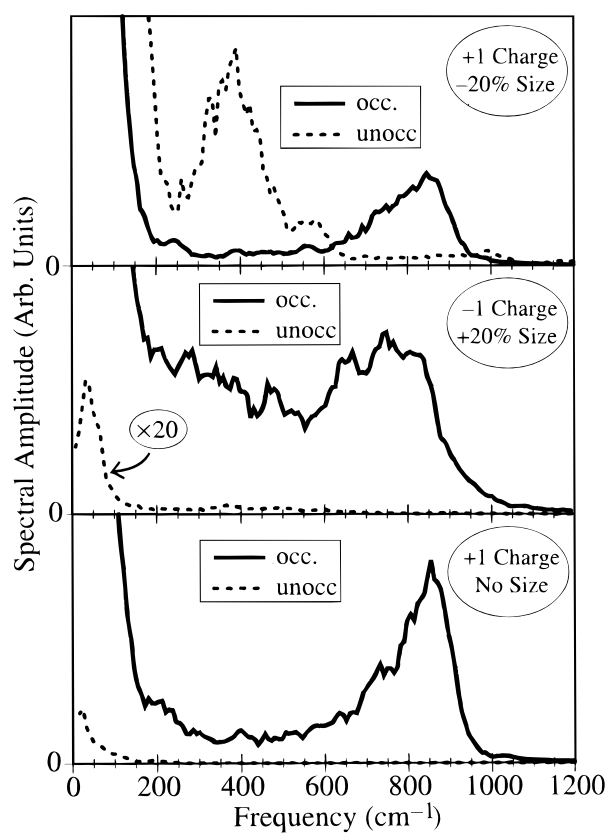

Figure 6. Influence spectra for the nonequilibrium dynamics of the individual unoccupied ground (dashed curves) and occupied excited (solid curves) energy levels for a variety of solute perturbations: positive change in charge with a simultaneous $20 \%$ size decrease (upper panel); negative change in charge with a simultaneous $20 \%$ size increase (center panel); and positive change in charge with no change in size (lower panel). These curves are Fourier transforms of the corresponding time-domain energy level curves presented in Figure 5.

curves in each panel show the evolution of the energy of the (unoccupied) neutral ground state as the solvent responds to the excitation of the solute; the difference between the two curves in each panel is the energy gap used to compute the corresponding $S(t)$ values in Figure 4. All three panels in Figure 5 are shown on the same absolute scale, so the magnitudes of the energy level changes for the different perturbations can be directly compared. The $y$ axes in the three panels in Figure 6, however, have been scaled relative to one another to emphasize the shapes of the spectra in each panel and thus are not comparable on an absolute scale. The most striking feature of Figures 5 and 6 is that the relaxation of the occupied state for each of the three perturbations is remarkably similar. This means that the impetus for solvating the excited state comes from the newly appeared charge on the solute; combining the charge change with either a positive or negative size change makes little difference in the driving force for relaxation of the new charge distribution. Thus, the differences in the overall solvent response associated with solute size changes, including the reasons for the breakdown of $\mathrm{LR}$, lie primarily in the dynamics of the unoccupied ground state.

In contrast to the relaxation of the occupied state, the unoccupied ground-state dynamics differ markedly depending on the size change of the solute. The reason for this variation involves a competition between different types of solvent translational motions: those responding to the solute's size change and those resulting from electrostriction. For the solute that is $20 \%$ smaller than the ground state and whose excited state has a +1 charge (Figures 5 and 6, upper panel), these motions must work cooperatively because the decreased size and the attractive ion-dipole forces work together to pull the first solvent shell in toward the solute. The net result is that the closest first-shell solvent molecules must translate inward by more than $20 \%$ of a solute radius to reestablish equilibrium around the charged excited-state solute, as discussed in more detail in the next section. This inward motion places these molecules far up the $1 / r^{12}$ repulsive core of the original ground state interaction potential, leading to a rise in the energy of the unoccupied state that is comparable to the magnitude of the relaxation of the excited state. It takes some time, however, for the first of these molecules to translate inward far enough to significantly affect the energy of the unoccupied state. Thus, the energy of the unoccupied state remains flat for $\sim 50 \mathrm{fs}$ before becoming destabilized by the presence of additional molecules inside the ground-state repulsive core (dotted curve, upper panel of Figure 5). The fact that different types of solvent motions are involved in the relaxation of the occupied and unoccupied states is emphasized in the upper panel of Figure 6. The solid curve makes clear that the relaxation of the occupied state is dominated by fast $\left(\sim 800 \mathrm{~cm}^{-1}\right)$ rotational motions as if only the charge had changed (cf. lower panel). The dotted curve shows that the destabilization of the unoccupied state is driven by slower $\left(\sim 300 \mathrm{~cm}^{-1}\right)$ solvent translational motions. In terms of the validity of LR, it is easy to see that this type of destabilization of the ground state is not represented by any of the solvent motions at equilibrium; $k T$ at room temperature is far too small for there to be any inward translational fluctuations of this magnitude. Thus, the breakdown of LR results from the fact that the solvent translational motions necessary to complete the nonequilibrium relaxation are simply not present at equilibrium.

The lower panels in Figures 5 and 6 show why LR works reasonably well for the case of purely dielectric solvation. As expected for the solvent response to a change in charge, the rapid inertial decay and subsequent oscillations in Figure 5 show that relaxation of the occupied state involves solvent librational motions, which also can be seen in the frequency domain in Figure 6 (solid curves). This reorientation of solvent molecules, however, has no effect on the energy of the uncharged ground state. Thus, the energy of the unoccupied ground state remains flat, except for the small "glitch" that occurs just after 200 fs (Figure 5, dotted curve). As documented previously by Rao and Berne $^{41}$ and also explored in the next section, this glitch results from the electrostriction associated with the charge change; it takes $\sim 200 \mathrm{fs}$ for the closest molecules to translate inward to establish the new local solvent structure, leading to a (relatively small) destabilization of the ground state. This delayed rise in the unoccupied state energy is manifested in the influence spectrum as a small peak at very low frequencies (dotted curve, Figure 6). Again, because of the steepness of the ground-state solute-solvent interaction potential, these types of inward translational fluctuations are not accessible at equilibrium. Thus, for the nonequilibrium dynamics, the destabilization of the unoccupied state results in an "extra" component in the relaxation of the energy gap that is not described by the fluctuations at equilibrium. This means that LR overestimates (albeit only slightly) the magnitude of the initial relaxation for the charge-only change. The overestimate arises because the LR prediction assumes that the motions available at equilibrium are all that are necessary for complete relaxation of the energy gap, but for the nonequilibrium dynamics, these motions provide only part of the total relaxation. The amount of the overestimate is the extra fraction of the final, nonequilibrium energy gap caused by the solvent translational motions that are not present at equilibrium. This idea that both solvent reorientation and electrostriction are important in ionic solvation is also consistent with theory. ${ }^{42}$

The center panels of Figures 5 and 6 show what happens for the case of a solute that gains an electron and undergoes a $20 \%$ 
increase in size. Here, the outward translational solvent motions that accompany the solute size increase are partially offset by the inward solvent motions associated with electrostriction. As a result, very little translational motion of the first solvent shell is needed to cause relaxation, as documented in more detail in the next section. Indeed, the dashed line in Figure 5 is flat (and the corresponding spectral amplitude in Figure 6 is extremely small), indicating that excitation has not changed the distance between the solute and the first solvent shell enough to significantly affect the energy of the unoccupied state. Thus, unlike the previous two cases, the slight nonlinearity in the solvent response for the -1 charge $/+20 \%$ size change does not result from the presence of nonequilibrium translational motions that affect the energy of the unoccupied state. Instead, the breakdown of LR arises for the same reason as that for the $+20 \%$ size-only increase discussed above: the outward motions necessary to accommodate the size change are assisted by translation-rotation coupling not present at equilibrium, as evident in the enhanced amplitude between 400 and $600 \mathrm{~cm}^{-1}$ in the excited-state influence spectrum in the center panel of Figure 6. A comparison of Figures 4 and 1, however, shows that the nonlinearity for the combined size-and-charge change is smaller than that for the size-only change. We identify two reasons for why the breakdown of LR is less severe for the combined size-and-charge change. First, for the combined change, the amount of outward translational motion needed to cause relaxation is partially balanced by electrostriction. This reduces the need for solvent translation relative to the size-only change, requiring less translation-rotation coupling. Second, a significant fraction of the solvation energy for the combined size-and-charge change results from the appearance of the charge. ${ }^{20}$ This means that solvent rotations, which, as discussed in the preceding paragraph, produce an essentially linear response, play an enhanced role in the relaxation for the combined change relative to the size-only change. In combination, the decreased emphasis of translational motions and the increased relative importance of solvent rotations result in a LR prediction that only slightly underestimates the rate of the inertial nonequilibrium relaxation, as observed in Figure 4.

Finally, it is worth emphasizing that the breakdown of LR described here for combined size-and-charge perturbations results directly from the steepness of the solute-solvent interaction potentials involved in mechanical solvation. Many authors have noted that response functions based on the equilibrium fluctuations of the excited-state solute energy level, rather than on the energy gap, often provide good predictions for nonequilibrium dynamics. ${ }^{2,5,9}$ The reasoning is that, unlike the equilibrium fluctuations that affect the energy gap, the fluctuations that affect just the excited state will capture more of the character of the solvent motions involved in the excitedstate nonequilibrium relaxation. Thus, use of excited-state equilibrium fluctuations to predict nonequilibrium dynamics works well for charge-only changes because the same librational motions involved both at equilibrium and during the nonequilibrium dynamics cause relaxation of the charged state without significantly affecting the neutral solute ground state. If a significant portion of the dynamics of the nonequilibrium energy gap comes from changes in the unoccupied-state energy, however, this type of argument is bound to fail. Figures 5 and 6 make clear that, for the realistic perturbations involving changes in both size and charge, neither the equilibrium fluctuations of the excited state nor those of the gap are sufficient for predicting the nonequilibrium dynamics. This is because the solvent motions that cause nonequilibrium relaxation are not

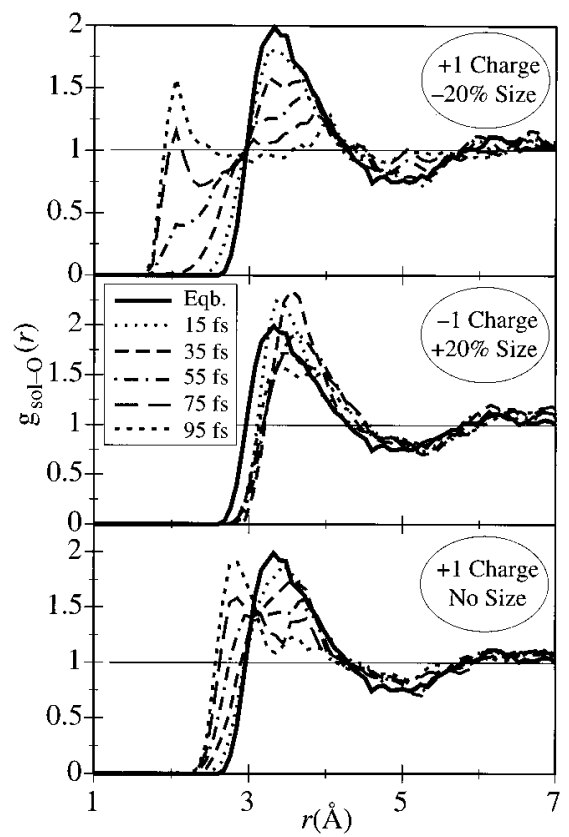

Figure 7. Time-dependent solute-solvent oxygen atom radial distribution functions $\left[g_{\text {sol-O }}(r)\right]$ showing the change in solvent structure following a variety of solute perturbations: positive change in charge with a simultaneous $20 \%$ size decrease (upper panel); negative change in charge with a simultaneous $20 \%$ size increase (center panel); and positive change in charge with no change in size (lower panel). Each curve is an average over $10 \mathrm{fs}$ of dynamics; see text for details. The heavy solid curve is the equilibrium radial distribution function; the dashed and dotted curves show the nonequilibrium radial distribution function at different times after excitation.

present at equilibrium. This is a violation of the LR approximation in the most basic sense: the perturbations involving size changes are "large" enough not to be describable by equilibrium solvent motions, even for the inertial dynamics.

C. Molecular Structure Evolution Associated with Nonpolar Solvation Dynamics. Throughout this paper, we have contended that, when the size and charge of a reactant change during an electron-transfer reaction, the different types of solvent motion present at equilibrium are not equally effective in relaxing and stabilizing the product. The spectral density analyses presented in Figures 2 and 6 argue that the solvent rotational motions present at equilibrium are effective in accommodating the new charge of the solute but that the solvent translational motions present at equilibrium are not effective at adapting to the new solute size. Although the spectral density analysis provides a great deal of insight, all of the conclusions rely on assumptions as to which specific solvent motions take place at particular frequencies. Thus, in this section, we take advantage of the molecular detail offered by nonequilibrium simulations to explore the way in which the local solvent structure evolves following changes in solute size and charge.

Figure 7 shows how the solute oxygen radial distribution function $\left[g_{\text {sol-O }}(r)\right]$ evolves for the first 100 fs following excitation for the same three perturbations considered in Figures 4-6. Each radial distribution function is computed by averaging 10 consecutive configurations per trajectory over the 100 nonequilibrium trajectories, for a total of 1000 configurations. For example, the dotted curves marked " $15 \mathrm{fs"} \mathrm{are} \mathrm{a} \mathrm{nonequi-}$ librium ensemble average of the dynamical configurations between 10 and $20 \mathrm{fs}$ after each perturbation. The solid curve in each panel shows $g_{\text {sol-O }}(r)$ for the equilibrium solute before excitation. Figure 8 shows the data of Figure 7 in a different way: the distance at which the radial distribution function has 


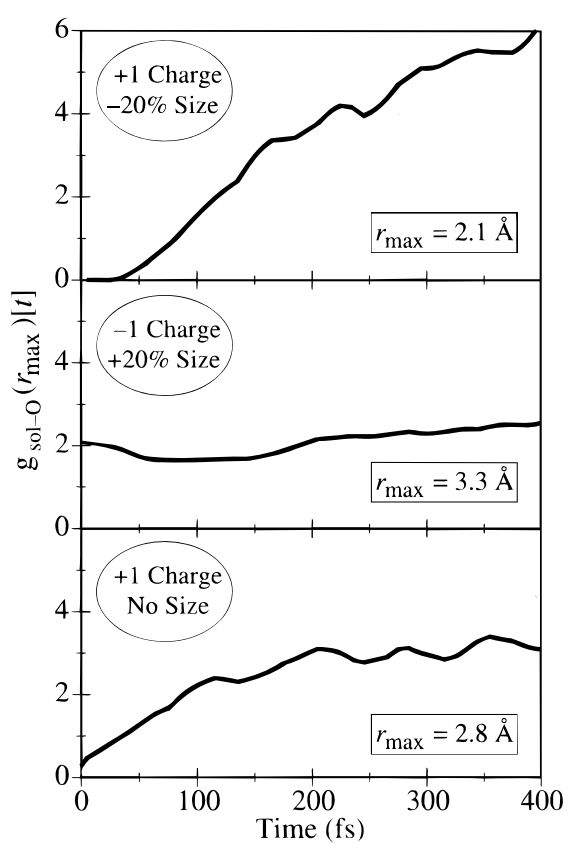

Figure 8. Time dependence of the value of the solute-solvent oxygen radial distribution function evaluated at the distance at which the function is a maximum after the excited-state relaxation is complete, $g_{\text {sol-O }}\left(r_{\max }\right)[t]$, for a variety of solute perturbations: positive change in charge with a simultaneous $20 \%$ size decrease (upper panel); negative change in charge with a simultaneous $20 \%$ size increase (center panel); and positive change in charge with no change in size (lower panel). The value of $r_{\max }$ for each case is shown; cf. the first $100 \mathrm{fs}$ of each curve to Figure 7.

its maximum value after the solvent has equilibrated around the excited-state solute, $r_{\max }$, is determined, and the value of the radial distribution function at this distance, $g\left(r_{\max }\right)$, is plotted as a function of time following excitation. Thus, each trace in Figure 8 shows how long it takes to reestablish the first solvent shell around the solute following each of the three perturbations.

The effects of electrostriction are most clearly seen in the lower panels of Figures 7 and 8, which show the evolution of $g_{\text {sol-O }}(r)$ resulting from the addition of charge to the solute. Immediately following excitation, Figure 7 shows that the first solvent shell moves inward toward the solute, with the closest solvent molecule [the "turn-on" point in $g(r)$ ] moving in from $2.7 \AA$ to a distance of $2.4 \AA$ after $\sim 100 \mathrm{fs}$, a finding similar to that of the previous work of Rao and Berne. ${ }^{41}$ This inward translational motion has little effect on the energy of the occupied state but causes a small destabilization of the ground state once the closest molecules have translated inside the repulsive part of the ground-state potential. Figure 8 shows that it takes $\sim 200$ fs before the new solvent structure around the charged solute is fully established, leading to the small delayed glitch in the unoccupied-state energy seen in Figure 5. Figure 7 also shows that, as far as the local solvent structure around the small solute is concerned, electrostriction is equivalent to a size decrease of $\sim 15 \%$. Thus, the destabilization of the ground state takes place slowly following excitation for exactly the same reasons that relaxation takes place slowly for a size-only decrease: there is only a small driving force for inward motion, and averaging over the phase makes the fastest inertial translational motions ineffective in promoting relaxation.

The upper panels in Figures 7 and 8 show what happens when the change in charge is accompanied by a $20 \%$ size decrease. For this case, the closest solvent molecules move inward from a distance of $\sim 2.7 \AA$ to within $1.8 \AA$ of the solute in the first 100 fs following excitation, an effective size decrease of $\sim 35 \%$.
This means that the solvent translational motions needed to accommodate the excited-state solute, both those resulting from the size decrease $(-20 \%)$ and those resulting from electrostriction (approximately $-15 \%$ ), are roughly additive. The fact that the closest first-shell molecules travel so far inward causes the large destabilization of the unoccupied ground state. Figure 8 shows that the distance the first-shell molecules need to translate is so large that essentially none of them have reached their new equilibrium position after 50 fs. Even after $400 \mathrm{fs}$, the new equilibrium structure is not completely established; as argued above, some of the molecules were moving the wrong way at the time of excitation. This leads to a relaxation of the energy gap that is much slower than that predicted by LR, as seen in Figure 4.

The center panels in Figures 7 and 8 show the local structural changes for the case in which the solute gains an electron and increases its size by $20 \%$. For this perturbation, the closest molecules move outward, from $\sim 2.7$ to $\sim 2.9 \AA$, in the first 100 fs following excitation. This is a net outward expansion of $\sim 7 \%$, again indicating a roughly additive relationship between the inward translational motions resulting from electrostriction (approximately $-15 \%$ ) and the outward motions resulting from the size increase $(+20 \%)$. The net increase in solute size means that there is a large force pushing the first-shell solvent molecules away from the solute. This force acts on the firstshell molecules whether they are moving in the correct direction or not, driving the first-shell molecules to rotate slightly to better fit within the second shell. Thus, the initial outward expansion of the first shell takes place quickly, as verified by both Figures 7 and 8 , so that translation-rotation coupling results in solvent relaxation that is only slightly faster than that predicted by LR (Figure 4). Because the translational motions for electrostriction and for the size increase are nearly balanced, however, relatively little net translation of the first solvent shell is required. This means that the final (radial) solvent structure is not very different from the initial equilibrium structure (Figure 7), so that the breakdown of the LR prediction is relatively minor.

Finally, Figure 9 shows the angular distribution of the firstshell solvent molecules around the solute as a function of time for the same perturbations studied in Figures $4-8$. The angle under consideration here is that between the water molecule dipole moment vector $(\mu)$ and the radial vector connecting the solute and solvent oxygen atom (r). The angular distributions are computed using only those solvent molecules within $5 \AA$ of the solute; thus, contributions are averaged only from the 12-18 closest molecules at each time step. Like the timedependent $g(r)$ values in Figure 7, each trace combines $10 \mathrm{fs}$ of dynamics per trajectory and is ensemble averaged over the 100 nonequilibrium trajectories. The heavy solid curve in each panel shows the equilibrium angular distribution around the neutral solute. The slight peak near $\cos (\mu \cdot \mathbf{r})=0.4$ in the equilibrium distribution is indicative of hydrophobic hydration; to maintain favorable hydrogen bonding, the first shell water molecules form a clathrate-type structure, directing their $\mathrm{H}$-bonds tangentially around the nonpolar ground-state solute.9,43 The changes in angular distribution with time seen in Figure 9 reflect the loss of hydrophobic hydration and the corresponding buildup of an ionic solvation structure around the newly charged solute.

The upper and lower panels of Figure 9 show the changes in the first-shell angular distribution when the solute gains a positive charge with (upper panel) or without (lower panel) an accompanying $20 \%$ size decrease. For both cases, the initial hydrophobic solvent structure is destroyed and the new firstshell ionic solvation structure, characterized by a peak near cos- 


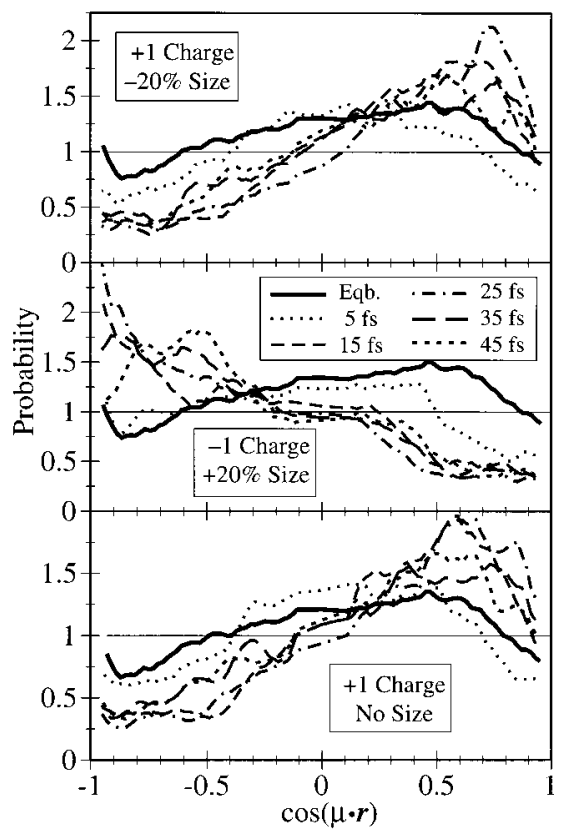

Figure 9. Time-dependent solvent angular distribution functions showing the change in solvent structure following a variety of solute perturbations: positive change in charge with a simultaneous $20 \%$ size decrease (upper panel); negative change in charge with a simultaneous $20 \%$ size increase (center panel); and positive change in charge with no change in size (lower panel). The relevant angle is that between the solvent dipole vector $(\mu)$ and the radial vector between the solute and solvent $(\boldsymbol{r})$. Each curve is an average over $10 \mathrm{fs}$ of dynamics for those solvent molecules within $5 \AA$ of the solute; see text for details. The heavy solid curve is the equilibrium angular distribution; the dashed and dotted curves show the nonequilibrium angular distribution at different times after excitation.

$(\mu \cdot \mathbf{r})=0.7$, is nearly fully developed within the first $50 \mathrm{fs}$ following excitation. The solvent molecule librational oscillations seen in Figures 4 and 5 are also visible in Figure 9; the probability of finding solvent molecules oriented with $\cos (\mu \cdot \mathbf{r})$ $=0.7$, for example, clearly oscillates in time. Overall, the angular relaxation of the first-shell solvent molecules is essentially identical for both the charge change and the combined charge change/size decrease. The similar relaxation dynamics in the two cases support the conclusions presented above in conjunction with Figure 6, namely, that the same solvent rotational motions are responsible for causing relaxation around a newly charged solute independent of whether the solute's size changes. This produces a similar relaxation of the occupied state for both cases; the differences in the relaxation of the energy gap result primarily from the way in which the corresponding change in size affects the unoccupied state (Figure 5).

The center panel in Figure 9 shows the time-dependent angular distribution for the first-shell solvent molecules when the solute becomes negatively charged and increases in size. There are two significant differences between this case and those shown in the upper and lower panels, in which the charge change is positive. First, the new ionic solvent structure for the negatively charged solute has the solvent $\mathrm{H}$ atoms pointing toward the solute, $\cos (\mu \cdot \mathbf{r})=-0.9$, instead of the other way around. Second, the disruption of the equilibrium hydrophobic solvent structure and the establishment of the new ionic solvation structure are slightly faster than those seen in the other two panels where there is a net size decrease. As discussed above, the expansion of the solute applies a large force to the firstshell molecules, forcing these molecules to rotate to better fit between the second-shell molecules to accommodate the new solute size. This large driving force causes the equilibrium solvent structure to dissipate in the first $10-20 \mathrm{fs}$ after excitation, leading to an overall relaxation that is slightly faster than that when there is a net inward solvent motion due to electrostriction or a solute size decrease. Overall, the data in Figures 7-9 support the conclusions drawn from Figures 4-6, verifying the usefulness of spectral density analysis for the study of solvation dynamics in water.

\section{Discussion}

In this paper, we have used molecular dynamics simulations to explore the reasons for the breakdown of the LR approximation in water for realistic perturbations that involve changes in solute size as well as charge. We find that LR predictions of mechanical solvation dynamics fail because the solvent translational motions involved in relaxing the excited-state solute are not present at equilibrium. For solute size increases, LR fails because the steepness of the solute-solvent interaction potential associated with the sudden expansion of the solute drives the first-shell solvent molecules into the second shell. The resulting relaxation takes advantage of translation-rotation coupling to utilize solvent motions that do not occur at equilibrium, resulting in faster solvation than that predicted by LR. For solute size decreases, on the other hand, the failure of LR results from the fact that relaxation requires motions of solvent molecules into the region formerly occupied by the ground-state solute. These motions destabilize the energy of the (unoccupied) ground state by an amount that is tens or hundreds of times larger than $k T$; there is no way these motions could be represented at equilibrium. Moreover, the solvent molecules that are most important in relaxation, those closest to the solute, are just as likely to be moving away from the solute as toward it at the time of excitation, leading to solvation dynamics that are much slower than those predicted by LR.

We also found that the nonlinearity of the solvent response can be exacerbated when the size and charge of the solute change simultaneously. Unlike translational motions, the rotational solvent motions that accompany solute charge changes are present at equilibrium, resulting in a solvent response that is nearly linear when solvent librations dominate the relaxation. The electrostriction resulting from a change in charge also requires solvent translational motions for relaxation; for the small solute considered here, these electrostrictive motions are equivalent to a $\sim 15 \%$ size decrease. For cases in which the size of the solute also changes, the solvent translational motions required to accommodate the new charge and new size of the solute turn out to be roughly additive. When the charge change is combined with a size increase, the effects of electrostriction and solute expansion nearly cancel, so that little net solvent translational motion is required. For this case, rotational motions dominate the solvent response, and LR would work reasonably well if the subtleties of the small outward solvent translational motions associated with the net size increase were ignored. For charge changes combined with solute size decreases, on the other hand, electrostriction mandates that additional inward solvent translations beyond those required for the size decrease are necessary to reestablish equilibrium. The requirement of additional solvent translational motions that are not present at equilibrium results in a massive breakdown of LR.

It is interesting to compare the breakdown of LR that we see for the combined size-and-charge changes to that observed in previous works. Ando and Kato have explored the aqueous solvation dynamics for a model of dimethylaniline in which both the charge distribution and the Lennard-Jones interaction potential changed upon excitation. ${ }^{12}$ Ando and Kato found, 
however, that the LR approximation worked remarkably well for their system. These authors noted that there was essentially no change in the local solvent structure upon excitation of their solute, ${ }^{12}$ a situation similar to our case of the solute that becomes negatively charged with a size increase. Thus, as argued above, because little solvent translation is required for relaxation, the resulting solvation dynamics fall within the linear regime. Other examples of nonlinear solvation dynamics have appeared in studies of solvent mixtures. ${ }^{7}$ In mixtures, the ground state of the solute is preferentially solvated by one component of the mixture, while the excited state may be preferentially solvated by the other component. This means that significant rearrangement of the first solvent shell is required to relax the excited state, requiring solvent motions that are not present at equilibrium.

Although we have focused the simulations in this paper on ionization of a neutral solute, many charge-transfer reactions involve further ionization or neutralization of already-charged solutes. The rough additivity of the solvent translational motions responding to solute size and charge changes allows us to make some general predictions regarding the solvent response for a wide variety of charge-transfer reactions. Consider the case of a reactant with a +1 charge that serves as the donor in an electron-transfer reaction, so that the product is a solute with a +2 charge that is slightly smaller in size than the reactant. The solvent around the reactant is already favorably oriented to accommodate a positive charge, so that solvent librational motions will play a lesser role in the relaxation when the charge is increased. Instead, we expect the bulk of the relaxation to result from inward solvent translational motions responding to both the increased electrostriction and the decreased size of the product. Thus, based on the above results, we anticipate slow solvent relaxation dynamics for this type of ionization. A similar prediction can be made for the case of a positively charged solute that is neutralized during the course of a reaction. Here, the loss of electrostriction is equivalent to a solute size increase, an effect that could be compounded by the fact that gaining an electron likely causes the neutral product to become larger than the parent cation. For this case, we would predict rapid solvent relaxation because of the large driving force for outward solvent translational motion. An MD simulation corresponding to exactly this case has already been done by Maroncelli and Fleming; ${ }^{9}$ indeed, Figure 20 of ref 9 shows that the nonequilibrium solvent response following neutralization of an ionized solute is significantly faster than the reverse reaction in which a neutral solute is ionized. Finally, recent work exploring the charge-transfer-to-solvent (CTTS) dynamics of the sodium anion in THF have found that the time scale for charge transfer is significantly slower than that for dielectric solvation. ${ }^{44}$ This is consistent with the idea of slow solvation resulting from the large size decrease of the solute associated with the detachment of the CTTS electron.

Even though the small solute in our simulations does not resemble the reactants studied in common charge-transfer systems, we expect its solvation behavior to be representative of small chemical groups in large molecules of interest. In our previous paper, we found that the solvent response for solute shape changes was nearly identical to that for changes in overall solute size. ${ }^{20}$ This is a direct consequence of the fact that motions of only one or two molecules are important in mechanical relaxation, so that an expansion of the solute in one direction behaves locally as if the entire solute had expanded by the same amount. Thus, because most charge-transfer reactions involve a change in the size or shape of the reactant, we expect that the observations made for the simple solute studied here will be applicable to electron-transfer reactions in more complex molecular systems. Of course, the mechanical interactions driving solvation dynamics for a local size change in a large molecule make up a much smaller fraction of the total solvation energy than those for a size change in the small atomic solutes considered here. This means that, even though we predict a breakdown of LR whenever size changes are involved, the failure of LR should become somewhat less severe in larger systems.

Finally, the nonlinearity of the solvent response has important implications for many previous studies of solvation dynamics in the literature. It is not clear, for example, that LR predictions should be used when studying the density dependence of nonpolar solvation dynamics; $; 25$ the nonlinear nature of nonpolar solvation dynamics may cause the nonequilibrium solvent relaxation to scale in a different way with density than would be predicted from equilibrium simulations. The nonlinearity associated with size changes also has implications for spectroscopic studies of solvation dynamics. The solvation dynamics that are measured in transient hole-burning or line-shape experiments result from the solvent fluctuations present at equilibrium for the solute in its ground state. Solvent response functions determined from the time-dependent fluorescence Stokes shift, ${ }^{2}$ on the other hand, are sensitive to the nonequilibrium relaxation of the energy gap following excitation of the solute. Photon-echo measurements involve an even more complex solvent response to the presence of an electronic coherence between the ground and excited electronic states of the solute. ${ }^{2,45}$ This means that, for photochemical reactions in which the reacting species undergo appreciable size changes, different types of experiments will measure different relaxation processes. Thus, it makes sense to use an equilibrium solvent response function to calculate a line shape ${ }^{22,23}$ and to use nonequilibrium solvation dynamics to understand time-dependent fluorescence experiments. ${ }^{14,46}$ It is not clear, however, that it is legitimate to compare the results of hole-burning experiments to time-dependent fluorescence (or photon-echo) experiments when changes in solute size are involved ${ }^{29-31}$ because of the intrinsic nonlinearity of the solvent response. Overall, given the general importance of solute size changes in chargetransfer reactions, theories of electron-transfer based on the LR approximation may not be valid. Moreover, when comparing different simulation and experimental results, care must be taken to ensure that the different methods under consideration are measuring the same solvent relaxation dynamics.

Acknowledgment. This work was supported by a National Science Foundation CAREER award under Grant CHE9733218. We also gratefully acknowledge the support of computational resources from the National Partnership for Advanced Computational Infrastructure (NPACI). Benjamin J. Schwartz is a Cottrell Scholar of Research Corporation and an Alfred P. Sloan Foundation Research Fellow.

\section{References and Notes}

(1) See, e.g.: Barbara, P. F.; Meyer T. J.; Ratner, M. A. J. Phys. Chem. 1996, 100, 13148. Hynes, J. T. In Ultrafast Dynamics of Chemical Systems Simon, J. D., Ed.; Kluwer Academic Publishers: Dordrecht, The Netherlands, 1994; Chapter 13, p 345.

(2) For some recent review articles, see: Maroncelli, M. J. Mol. Liq 1993, 57, 1. Cho, M.; Fleming, G. R. Annu. Rev. Phys. Chem. 1996, 47, 109. Rossky, P. J.; Simon, J. D. Nature, 1994, 370, 263. DeBoeij, W. P.; Pshenichnikov, M. S.; Wiersma, D. A. Annu. Rev. Phys. Chem. 1998, 49, 99. Stratt, R. M.; Maroncelli, M. J. Phys. Chem. 1996, 100, 12981.

(3) Allen, M. P.; Tildesely, D. J. Computer Simulation of Liquids; Oxford University Press: New York, 1987. 
(4) Chandler, D. Introduction to Statistical Mechanics; Oxford University Press: New York, 1989; Chapter 8.

(5) Fonseca, T.; Ladanyi, B. M. J. Mol. Liq. 1994, 60, 1. Fonseca, T.; Ladanyi, B. J. Phys. Chem. 1991, 95, 2116.

(6) Skaf, M. S.; Ladanyi, B. M. J. Phys. Chem. 1996, 100, 18258.

(7) Cichos, F.; Brown, R.; Rempel, U.; von Borczyskowsi, C. J. Phys. Chem. A 1999, 103, 2506. Day, T. J. F.; Patey, G. N. J. Chem. Phys. 1997, 106, 2782. Laria, D.; Skaf, M. S. J. Chem. Phys. 1999, 111, 300.

(8) Phelps, D. K.; Weaver, M. J.; Ladanyi, B. M. Chem. Phys. 1993, 176,575 .

(9) Maroncelli, M.; Fleming, G. R. J. Chem. Phys. 1988, 89, 5044.

(10) Bader, J.; Chandler, D. Chem. Phys. Lett. 1989, 157, 501.

(11) Brown, R. J. Chem. Phys. 1995, 102, 9059. Muiño, P. L.; Callis,

P. R. J. Chem. Phys. 1994, 100, 4093.

(12) Ando, K.; Kato, S. J. Chem. Phys. 1991, 95, 5966.

(13) Maroncelli, M. J. Chem. Phys. 1991, 94, 2084.

(14) Carter, E. A.; Hynes, J. T. J. Chem. Phys. 1991, 94, 5961.

(15) Re, M.; Laria, D. J. Phys. Chem. B 1997, 101, 10494.

(16) Schwartz, B. J.; Rossky, P. J. J. Chem. Phys. 1994, 101, 6902.

(17) Ladanyi, B. M.; Stratt, R. M. J. Phys. Chem. 1995, 99, 2502.

Ladanyi, B. M.; Stratt, R. M. J. Phys. Chem. 1996, 100, 1266.

(18) Kalbfleisch, T. S.; Ziegler, L. D.; Keyes, T. J. Chem. Phys. 1996, 105,7034

(19) Kumar, P. V.; Maroncelli, M. J. Chem. Phys. 1995, 103, 3038. Ladanyi, B. M.; Maroncelli, M. J. Chem. Phys. 1998, 109, 3204.

(20) Tran, V.; Schwartz, B. J. J. Phys. Chem. B 1999, 103, 5570.

(21) Ladanyi, B. M.; Stratt, R. M. J. Phys. Chem. A 1998, 102, 1068.

(22) Stephens, M. D.; Saven, J. G.; Skinner, J. L. J. Chem. Phys. 1997, $106,2129$.

(23) Saven, J. G.; Skinner, J. L. J. Chem. Phys. 1993, 99, 4391.

(24) Herman, M. F.; Berne, B. J. J. Chem. Phys. 1983, 78, 4103. 4169 .

(25) Yamaguchi, T.; Kimura, Y.; Hirota, N. J. Chem. Phys. 1999, 111,

(26) Maroncelli, M.; Kumar, P. V.; Papazyan, A.; Horng, M. L.; Rosenthal, S. J.; Fleming, G. R. In Ultrafast Reaction Dynamics and Solvent Effects; Gauduel, Y., Rossky, P. J., Eds.; AIP Conference Proceedings; American Institute of Physics: Melville, NY, 1994; No. 298, p 310.

(27) Jimenez, R.; Fleming, G. R.; Kumar, P. V.; Maroncelli, M. Nature 1994, 369, 471. Lang, M. J.; Jordanides, X. J.; Song, X.; Fleming, G. R. J. Chem. Phys. 1999, 110, 5884.

(28) Rosenthal, S. J.; Xie, X. L.; Du, M.; Fleming, G. R. J. Chem. Phys. 1991, 95, 4715 .

(29) See, e.g.: Berg, M. Chem. Phys. Lett. 1994, 228, 317. Bhattacharya,
S.; Bagchi, B. J. Chem. Phys. 1998, 109, 7885. Berg, M. J. Phys. Chem. A 1998, 102, 17. Berg, M. J. Chem. Phys. 1999, 110, 8577.

(30) See, e.g.: Fourkas, J. T.; Berg, M. J. Chem. Phys. 1993, 98, 7773. Wendt, H.; Richert, R. J. Phys. Chem. A 1998, 102, 5775.

(31) Ma, J.; Vanden Bout, D.; Berg, M. J. Chem. Phys. 1995, 103, 9146.

(32) Schwartz, B. J.; Rossky, P. J. J. Mol. Liq. 1995, 65, 23. Schwartz,

B. J.; Rossky, P. J. J. Chem. Phys. 1996, 105, 6997 and references therein

(33) See, e.g.: Hasted, J. B.; Husain, S. K.; Frescura, F. A. M.; Birch, J. R. Chem. Phys. Lett. 1985, 118, 622. Castner, E. W.; Chang, Y. J.; Chu, Y. C.; Walrafen, G. E. J. Chem. Phys. 1995, 102, 653. Zolotov, B.; Gan, A.; Fainberg, B. D.; Huppert, D. Chem. Phys. Lett. 1997, 265, 418. Palese, S.; Schilling, L.; Miller, R. J. D.; Staver, P. R. J. Phys. Chem. 1994, 98, 6308. Walrafen, G. E. J. Phys. Chem. 1990, 94, 2237. Bursulaya, B. D.; Kim, H. J. J. Chem. Phys. 1998, 109, 4911. Sastry, S.; Stanley, H. E.; Sciortino, F. J. Chem. Phys. 1994, 100, 5361. Madden, P. A.; Impey, R. W. Chem. Phys. Lett. 1986, 123, 502. Cho, M.; Fleming, G. R.; Saito, S.; Ohmine, I.; Stratt, R. M. J. Chem. Phys. 1994, 100, 6672.

(34) Toukan, K.; Rahman, A. Phys. Rev. B 1985, 31, 2643.

(35) Berendsen, H. J. C.; Potsma, J. P. M.; van Gunsteren, W. F. Hermans, J. In Intermolecular Forces; Pullman, B., Ed.; D. Reidel: Dordrecht, The Netherlands, 1991; p 331.

(36) See, e.g.: Watanabe, K.; Klein, M. L. Chem. Phys. 1989, 131, 157. Barrat, J.-L.; McDonald, I. R. Mol. Phys. 1990, 70, 535. Wallqvist, A.; Teleman, O. Mol. Phys. 1991, 74, 515

(37) Steinhauser, O. Mol. Phys. 1982, 45, 335

(38) A detailed statistical argument in favor of this choice is given in Schwartz, B. J.; Rossky, P. J. J. Chem. Phys. 1994, 101, 6917.

(39) Nowak, R.; Bernstein, E. R. J. Chem. Phys. 1987, 86, 4783. See also refs $29-31$.

(40) This is readily apparent from any table of ionic or van der Waals radii. See, e.g.: Huheey, J. E Inorganic Chemistry, $3^{\text {rd }}$ ed.; Harper \& Row: New York, 1983.

(41) Rao, M.; Berne, B. J. J. Phys. Chem. 1981, 85, 1498.

(42) Yoshimori, A.; Day, T. F. J.; Patey, G. N. J. Chem. Phys. 1998 , 109,3222

(43) See, e.g.: Pangali, C.; Rao, M.; Berne, B. J. J. Chem. Phys. 1979, 71, 2975. Heinzinger, K.; Palinkas, G. In The Chemical Physics of Solvation; Dogonadze, R. R., Ed.; Elsevier: Amsterdam, 1985; Part A, p 313.

(44) Barthel, E. R.; Martini, I.; Schwartz, B. J. J. Chem. Phys. 2000, in press.

(45) See, e.g.: Shemetulskis, N. E.; Loring, R. F. J. Chem. Phys. 1992 97, 1217.

(46) Schwartz, B. J.; Rossky, P. J. J. Phys. Chem. 1995, 99, 2953. 